\title{
Scattering Analysis of a Compact Dipole Array with Series and Parallel Feed Network including Mutual Coupling Effect
}

\author{
H. L. Sneha, Hema Singh, and R. M. Jha \\ Computational Electromagnetics Laboratory, CSIR-National Aerospace Laboratories, Bangalore 560017, India \\ Correspondence should be addressed to Hema Singh; ishihema30@gmail.com
}

Received 28 January 2013; Revised 30 April 2013; Accepted 7 May 2013

Academic Editor: Hon Tat Hui

Copyright (C) 2013 H. L. Sneha et al. This is an open access article distributed under the Creative Commons Attribution License, which permits unrestricted use, distribution, and reproduction in any medium, provided the original work is properly cited.

\begin{abstract}
The current focus in defense arena is towards the stealth technology with an emphasis to control the radar cross-section (RCS). The scattering from the antennas mounted over the platform is of prime importance especially for a low-observable aerospace vehicle. This paper presents the analysis of the scattering cross section of a uniformly spaced linear dipole array. Two types of feed networks, that is, series and parallel feed networks, are considered. The total RCS of phased array with either kind of feed network is obtained by following the signal as it enters through the aperture and travels through the feed network. The RCS estimation of array is done including the mutual coupling effect between the dipole elements in three configurations, that is, side-by-side, collinear, and parallel-in-echelon. The results presented can be useful while designing a phased array with optimum performance towards low observability.
\end{abstract}

\section{Introduction}

The radar cross-section (RCS) is essentially a measure of electric field scattered from an object towards the radar [1]. A phased array antenna system mounted on the target scatters the incident signals, contributing significantly towards the total RCS of the target. The antenna scattering is function of parameters like antenna type [2], array configuration, and the nature of feed network. In addition, the presence of mutual coupling in between the antenna elements plays an important role in overall RCS. The emerging stealth technologies aim at reducing the scattering from an object to the least possible extent in order to shield it from the enemy radars. However, low observable platform has consequence of sacrificing array performance in terms of gain and radiation characteristics [3].

In the open domain, several techniques have been proposed for the estimation of the antenna RCS. These include numerical techniques [4] as well as high-frequency techniques [5], along with the optimization [6]. Most of the approaches neglect the presence of mutual coupling, edge effects, and the higher order reflections.
Most of the proposed methods focus on the compensation of mutual coupling in receiving arrays $[7,8]$ and the array feed network [9-12]. The behavior of antenna arrays in receiving and transmitting modes needs not be same. The factors like different signal paths within the transmitting and receiving array system and different antenna excitations in the two systems make the mutual coupling effect different in the two modes [13]. The techniques of estimating the mutual impedance for transmitting arrays like conventional impedance matrix method [14] differ from those for receiving array. The techniques to calculate the receiving mode impedance include full-wave methods [15, $16]$, the calibration methods $[17,18]$, and the receivingmutual impedance method (RMIM) [19-21]. The efficiency and accuracy of receiving mutual impedances is further improved by preserving the original boundary conditions of the compact array during the analysis [22]. Niow et al. [23] suggested the method for the compensation of mutual coupling in transmitting arrays. The compensation network, designed in terms of mutual impedances, is employed to predict the radiation pattern using the principle of pattern multiplication. This approach facilitates the port decoupling 


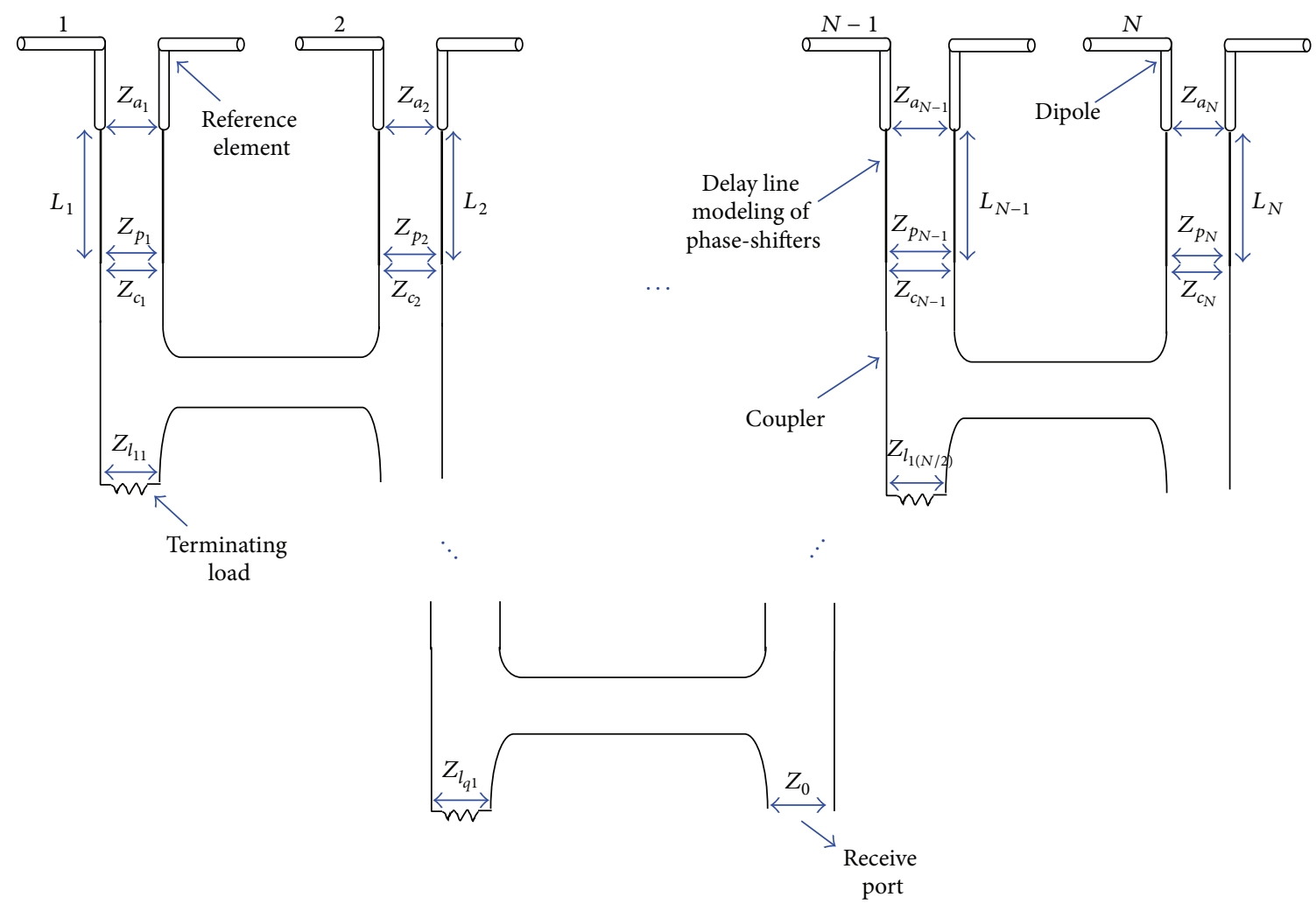

FIgURE 1: Typical parallel-feed network of dipole phased array.

and design of matching circuits towards the maximum power transfer. Wang and Hui [24] proposed a system identification method for the wideband mutual coupling compensation of receiving arrays. A multiport compensation network is obtained based on the receiving mutual impedances of an antenna array over the frequency band.

The effect of feed network on the RCS of the phased array has been analyzed $[9,10]$ using analytical technique of tracing the signal path through the antenna system. However, the proposed method considered an array of identical isotropic elements. The mutual coupling, edge effects, and the higher order reflections were not taken into account. In this paper, an attempt is made to analyze the antenna mode scattering of a linear series-fed [25] and parallel-fed dipole array (Figure 1), considering the mutual coupling effect. The variation in the array configuration (side-by-side, collinear, and parallel-inechelon) and the type of amplitude distribution (uniform unit amplitude, Taylor, cosine-squared on a pedestal, and DolphChebyshev) are studied. The RCS formulation involves the tracing of the signal path from the antenna aperture into the antenna system for estimation of the scattered field at each component level. The individual contributions towards the scattered field are expressed in terms of the reflection and transmission coefficients of components in the antenna system. These scattered fields at each level of antenna system are then coherently summed up to arrive at the total antenna array RCS.
Section 2 presents a brief analysis on the RCS formulation of linear dipole arrays with different feed network in the presence of mutual coupling. The simulation results comparing the RCS of dipole array with and without mutual coupling are discussed in Section 3. The role of the optimum load in the feed network in RCS estimation is analyzed.

\section{RCS Formulation for a Linear Dipole Array}

The RCS of an object for a plane wave incidence can be expressed as the ratio of the scattered field to the incident field. The antenna mode scattered field is expressed as [26]

$$
\vec{E}_{n}^{s}(\theta, \phi)=\left[\frac{j \eta}{4 \lambda Z_{a}} \vec{h}\left\{\vec{h} \cdot \vec{E}^{i}(\theta, \phi)\right\} \frac{e^{-j \vec{k} \vec{R}}}{R}\right] \Gamma_{n}^{r}(\theta, \phi),
$$

where $\vec{E}_{n}^{s}$ is the scattered field at the $n$th element, $\vec{E}^{i}$ is the incident field, $(\theta, \phi)$ is the direction of signal, $Z_{a}$ is the radiation impedance, $\lambda$ is the wavelength, $\eta$ is the impedance of medium surrounding the antenna, $\vec{k}$ is the wave vector, $R$ is the distance between the target and the observation point, $\Gamma_{n}^{r}$ is the total reflected signal returning to aperture element $n$, and $\vec{h}$ is the effective height of the antenna element. For a 


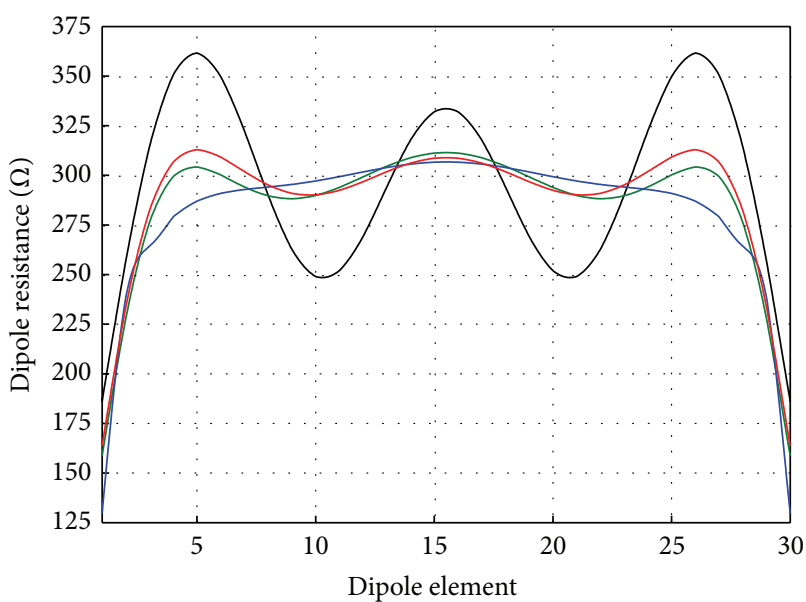

$\begin{array}{ll}\text { — Uniform unit amplitude } & \text { - Dolph-Chebyshev } \\ \text { Cosine squared on a pedestal } & \text { Taylor }\end{array}$

(a) Resistance

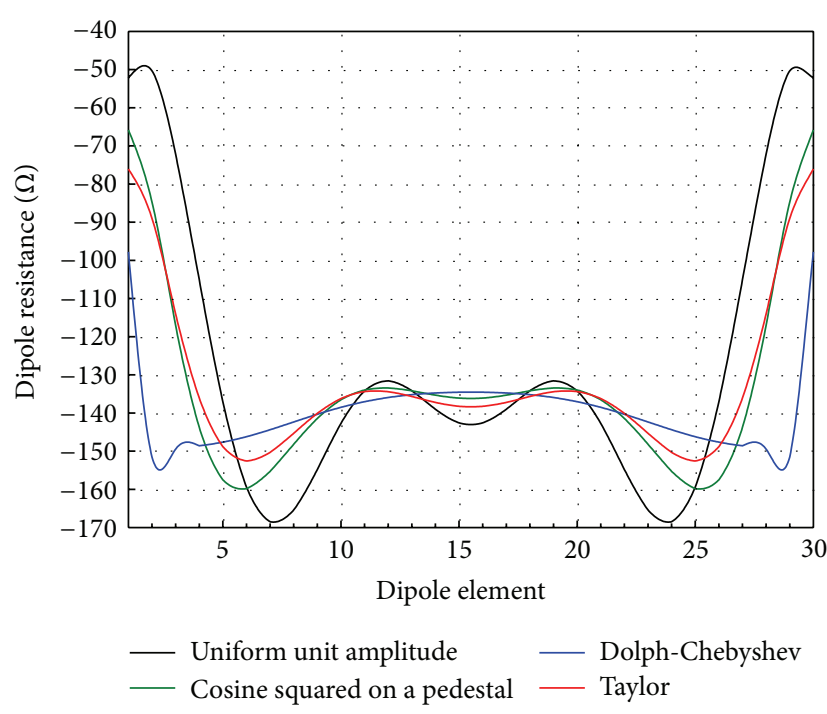

(b) Reactance

FIGURE 2: Terminal impedance $Z_{a_{n}}$ of the dipole elements in side-by-side configuration.

unit amplitude plane wave impinging on the array of lossless $x$-polarized dipoles placed in free space, (1) becomes

$$
\vec{E}_{n}^{s}(\theta, \phi)=\left[\frac{j \eta_{o}}{4 \lambda Z_{a}} h^{2}(\widehat{x} \cdot \widehat{\theta}) e^{-j \vec{k} \cdot \vec{d}_{n}} \frac{e^{-j \vec{k} \vec{R}}}{R}\right] \Gamma_{n}^{r}(\theta, \phi) \hat{x},
$$

where $\eta_{0}$ is the free space impedance and $\vec{d}_{n}$ is the distance vector. Assuming a uniform dipole array with interelement spacing $d$ along $x$-axis, the total scattered field is given by

$$
\sum_{n=1}^{N} \vec{E}_{n}^{s}(\theta, \phi)=\sum_{n=1}^{N}\left\{\frac{j \eta_{o}}{4 \lambda Z_{a}} h^{2} \cos \theta \Gamma_{n}^{r}(\theta, \phi) e^{j(n-1) \alpha} \frac{e^{-j \vec{k} \vec{R}}}{R} \hat{x}\right\},
$$

where $\alpha=k d \sin \theta \cos \phi$ represents the interelement space delay of the wave incident along $x$-direction; $k$ is the wave number.

The total RCS of the array expressed in terms of (3) is

$$
\begin{aligned}
\sigma(\theta, \phi) & =\lim _{R \rightarrow \infty} 4 \pi R^{2} \frac{\left|\sum_{n=1}^{N} \vec{E}_{n}^{s}(\theta, \phi)\right|^{2}}{\left|\vec{E}^{i}(\theta, \phi)\right|^{2}} \\
& =4 \pi\left|\sum_{n=1}^{N}\left\{\frac{j \eta_{o}}{4 \lambda Z_{a}} h^{2} \cos \theta \vec{E}_{n}^{r}(\theta, \phi)\right\}\right|^{2},
\end{aligned}
$$

where $\vec{E}_{n}^{r}(\theta, \phi)=\Gamma_{n}^{r}(\theta, \phi) e^{j(n-1) \alpha}$ represents the total scattered field returning to the aperture. This can be expressed as the summation of individual scattering sources of the feed network by navigating through the signal as it moves towards the receive port.

In general, a typical feed network will comprise of antenna elements, phase-shifters, couplers, and the terminating loads [27]. Although an efficient receiver is intended to receive the entire power incident on it, certain amount of signal will be lost due to scattering within the antenna system at components level before reaching the receive port. This scattered field is determined by the magnitude of reflection and transmission coefficients at different levels of feed network, which are due to the impedance mismatches prevailing at their junctions. However, it is to be noted that the expressions presented in this paper assume reciprocal feed network components and neglect the effect of higher order scattering.

The first level of mismatch experienced by the incoming signal will be at the junction of dipole terminals and the phase-shifters. At this level, the reflection coefficient of dipole element is given by [25]

$$
r_{r_{n}}=\left|\frac{Z_{a_{n}}-Z_{0}}{Z_{a_{n}}+Z_{0}}\right|
$$

where $r_{r_{n}}$ is the reflection coefficient of the $n$th dipole, $Z_{a_{n}}$ is the terminal impedance of the $n$th dipole, and $Z_{0}$ is the characteristic impedance of the delay line modeled phaseshifter.

The terminal impedance of the dipoles is function of both amplitude distribution and the array configuration [26]. Figures 2, 3, and 4 indicate the variation of the impedance at the terminals of dipoles for different combinations of feed current and the array geometry. The choice of array configuration controls the antenna impedance that will further have an impact on the scattered field and RCS due to radiators; that is,

$$
\begin{gathered}
\vec{E}_{r_{n}}^{r}(\theta, \phi)=r_{r_{n}} e^{j 2(n-1) \alpha}, \\
\sigma_{r}(\theta, \phi)=\left|\sum_{n=1}^{N}\left\{\frac{j \eta_{o}}{4 \lambda Z_{a}} h^{2} \cos \theta\left(r_{r_{n}} e^{j 2(n-1) \alpha}\right)\right\}\right|^{2} .
\end{gathered}
$$




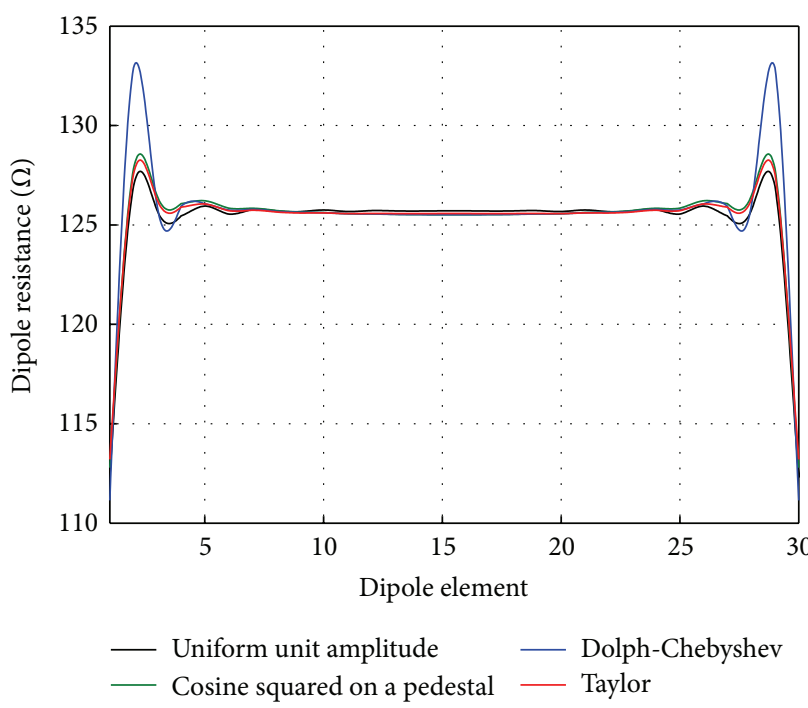

(a) Resistance

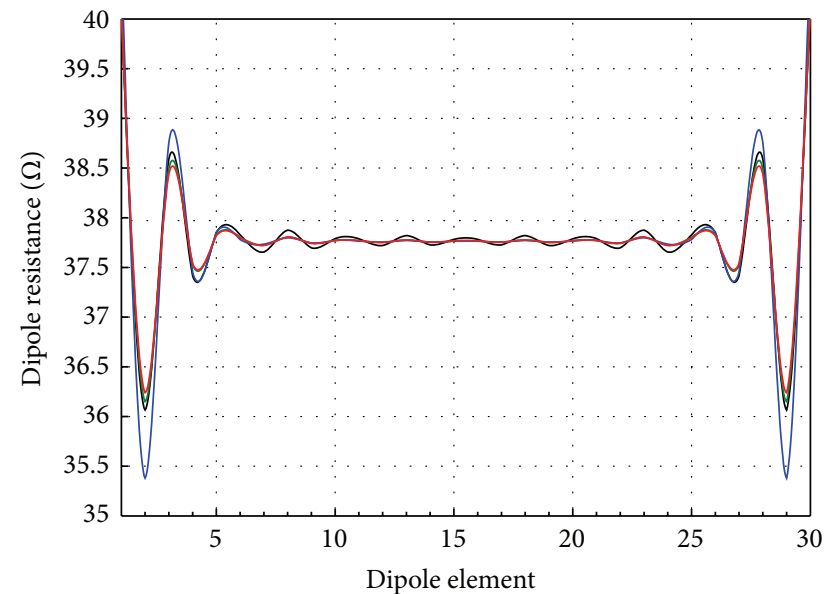

$\begin{array}{ll}\text { — Uniform unit amplitude } & \text { - Dolph-Chebyshev } \\ \text { Cosine squared on a pedestal } & \text { Taylor }\end{array}$

(b) Reactance

FIGURE 3: Terminal impedance $Z_{a_{n}}$ of the dipole elements in collinear configuration.

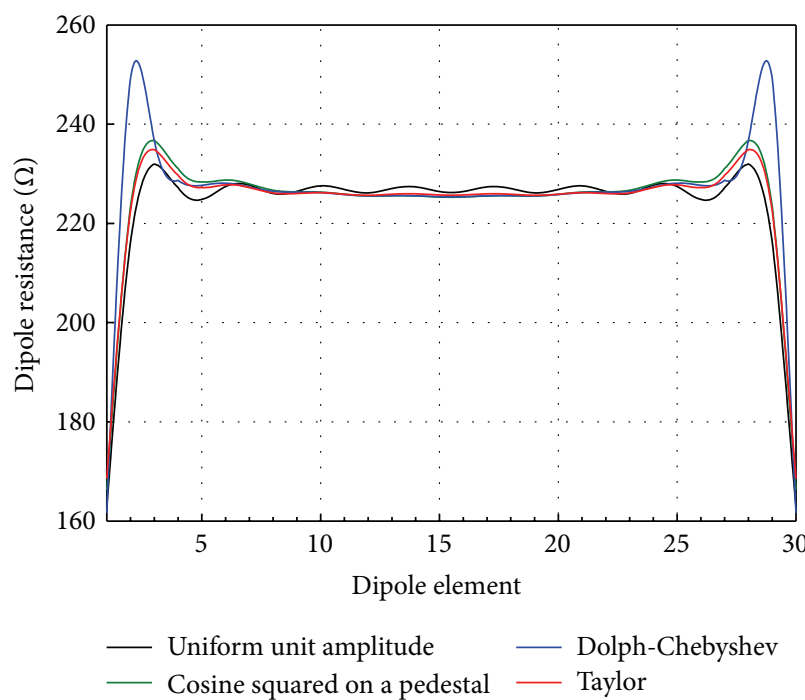

(a) Resistance

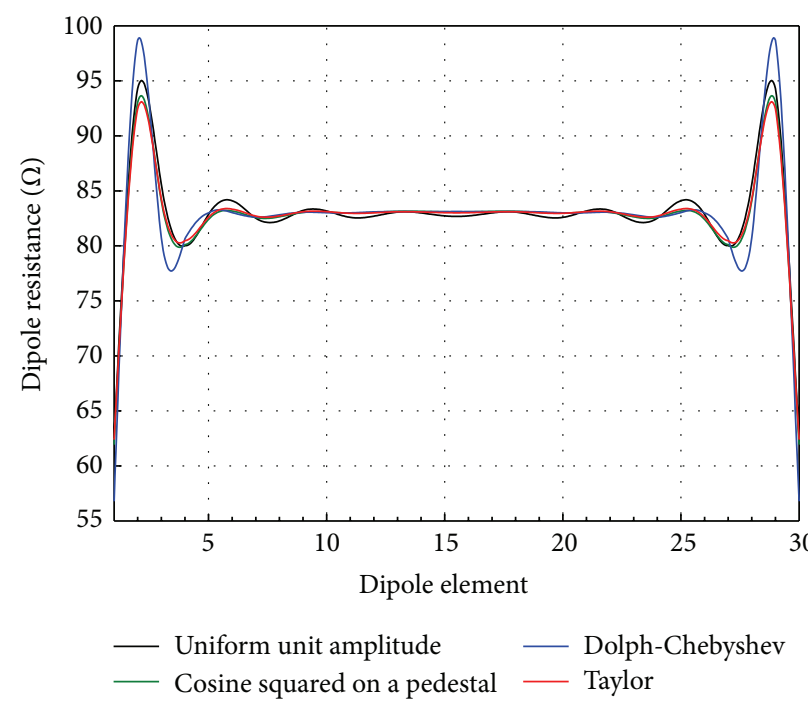

(b) Reactance

FIGURE 4: Terminal impedance $Z_{a_{n}}$ of the dipole elements in parallel-in-echelon configuration.

Following a similar analysis, one gets the scattered field and the RCS due to the mismatch at the phase-shifters

$$
\begin{gathered}
\vec{E}_{p_{n}}^{r}(\theta, \phi)=t_{r_{n}}^{2} r_{p_{n}} e^{j 2(n-1) \alpha} ; \quad r_{p_{n}}=\left|\frac{Z_{p_{n}}-Z_{0}}{Z_{p_{n}}+Z_{0}}\right|, \\
\sigma_{p}(\theta, \phi)=\left|\sum_{n=1}^{N}\left\{\frac{j \eta_{o}}{4 \lambda Z_{a}} h^{2} \cos \theta\left(t_{r_{n}}^{2} r_{p_{n}} e^{j 2(n-1) \alpha}\right)\right\}\right|^{2},
\end{gathered}
$$

where $r_{p_{n}}$ is the reflection coefficient at the phase-shifters, $t_{r_{n}}$ is the transmission coefficient of the radiating elements, and
$Z_{p_{n}}$ is the translated dipole terminal impedance as viewed at the end of phase-shifters. Further, the signal might suffer from reflection due to the differing impedances of phaseshifter terminals and the coupler ports. The scattering at this level is determined by the nature of couplers and is thus specific to the type of feed network exciting the dipoles.

2.1. Dipole Array with Series Feed Network. In this paper, the dipole elements in the series-fed linear phased array are excited using lossless four-port couplers (Figure 5). Port 1 and Port 2 of each coupler are taken as a part of the main feed line, while Port 3 and Port 4 are connected to the dipoles via 


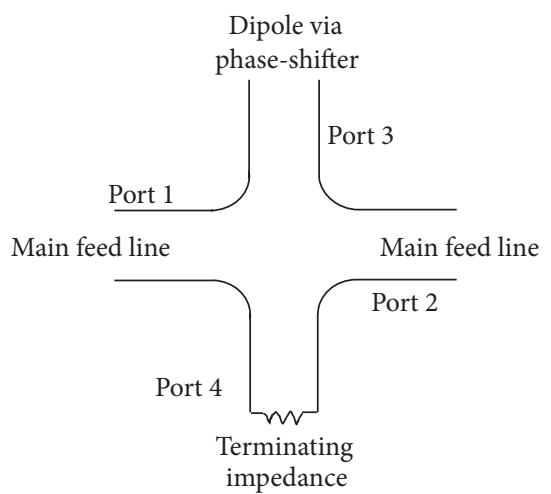

FIGURE 5: Schematic of a four-port coupler of the series feed network in phased array.

phase-shifters and the terminating load, respectively. Further, Port 1 of the first element of the array acts as the receiving port while Port 2 of the last element is terminated with a matched load. As a result, Port 1 and Port 2 serve as input and output, while Port 3 and Port 4 possess coupling and isolating characteristics. Moreover, Ports $(1,4)$ and $(2,3)$ constitute isolated port pairs.

The coupling and the transmission coefficients of the coupler ports, $j c_{n}$ and $t_{c_{n}}$, in the presence of mutual coupling are given by [25]

$$
\begin{gathered}
c_{n}=\frac{Z_{a_{n}} i_{n}^{2}}{\sum_{p=1}^{N} Z_{a_{p}} i_{p}^{2}-\sum_{q=1}^{n-1} Z_{a_{q}} i_{q}^{2}}, \\
t_{c_{n}}^{2}=1-c_{n}^{2},
\end{gathered}
$$

where $i_{n}$ is the current at the feed terminals of the $n$th antenna element.

Equations (8a) and (8b) indicate that the values of coupling and transmission coefficients depend on the terminal impedance of dipoles and the type of the amplitude distribution.

In other words, the coupling coefficients of the couplers in the feed network are function of both amplitude distribution and array configuration (Figures 6, 7, and 8).

In general, the scattered field and the RCS of $n$th dipole element due to the mismatch at the coupling port of couplers are expressed as

$$
\begin{gathered}
\vec{E}_{c_{n}}^{r}(\theta, \phi)=t_{r_{n}}^{2} t_{p_{n}}^{2} r_{c_{n}} e^{j 2(n-1)\left(\alpha+\alpha_{s}\right)}, \\
\sigma_{c}(\theta, \phi)=\left|\sum_{n=1}^{N}\left\{\frac{j \eta_{o}}{4 \lambda Z_{a}} h^{2} \cos \theta\left(t_{r_{n}}^{2} t_{p_{n}}^{2} r_{c_{n}} e^{j 2(n-1)\left(\alpha+\alpha_{s}\right)}\right)\right\}\right|^{2},
\end{gathered}
$$

where $r_{c_{n}}=\left|\left(Z_{c_{n}}-Z_{p_{n}}\right) /\left(Z_{c_{n}}+Z_{p_{n}}\right)\right|$ is the reflection coefficient at the couplers, $Z_{c_{n}}$ is the impedance at the coupling port of

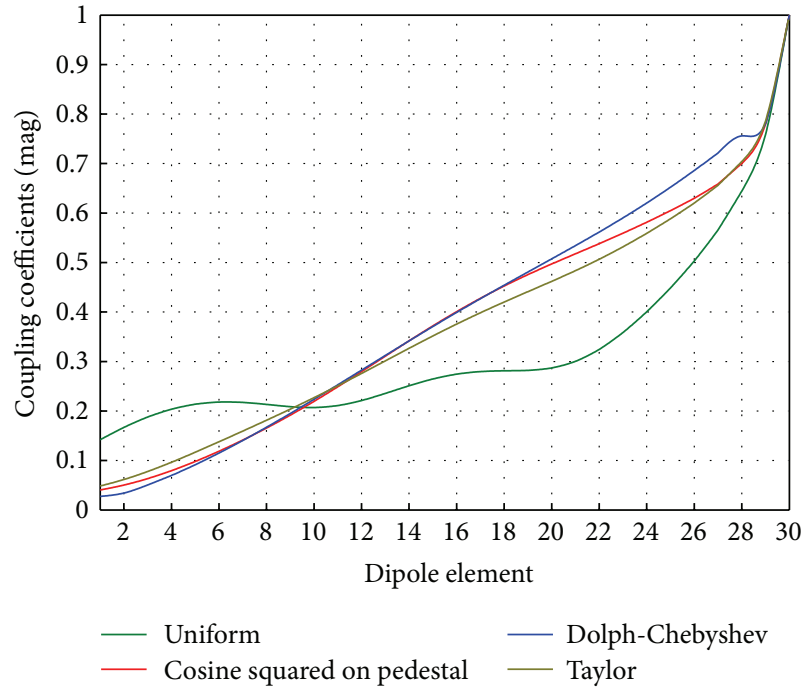

FIGURE 6: Coupling coefficients for different amplitude distributions in a series-fed linear dipole array; side-by-side configuration. $d=$ $0.1 \lambda$.

the couplers, $\alpha_{s}=k d \sin \theta_{s} \cos \phi_{s}$ is the interelement phase to scan the antenna beam along $x$-axis at $\left(\theta_{s}, \phi_{s}\right)$, and $t_{p_{n}}$ is the transmission coefficient of the phase-shifters.

Beyond coupling port of the coupler in series feed network, there can be several possible paths for the signal to travel further into the antenna system. It can either (i) move towards the previous element and/or next element and/or its own (self) terminating load and/or (ii) retrace its own path. The scattered fields corresponding to each of the signal paths are given by [23]

$$
\begin{aligned}
& \vec{E}_{1_{n}}^{r}(\theta, \phi)=\left[t_{r_{n}} t_{p_{n}} r_{l_{n}} j c_{n} e^{j(n-1)\left(\alpha+\alpha_{s}\right)}\right. \\
& \left.\times \sum_{m=n+1}^{N}\left(t_{r_{m}} t_{p_{m}} j c_{m} e^{j(m-1) \zeta} \prod_{i=n}^{m-1} t_{c_{i}} e^{j \psi}\right)\right], \\
& \vec{E}_{2_{n}}^{r}(\theta, \phi)=\left[t_{r_{n}} t_{p_{n}} j c_{n} e^{j(n-1)\left(\alpha+\alpha_{s}\right)}\right. \\
& \left.\times \sum_{m=1}^{n-1} t_{r_{m}} t_{p_{m}} r_{l_{m}} j c_{m} e^{j(m-1) \zeta} \prod_{i=m}^{n-1} t_{c_{i}} e^{j \psi}\right], \\
& \vec{E}_{3_{n}}^{r}(\theta, \phi)=r_{l_{n}} t_{r_{n}}^{2} t_{p_{n}}^{2} t_{c_{n}}^{2} e^{j 2(n-1)\left(\alpha+\alpha_{s}\right)}, \\
& \vec{E}_{4_{n}}^{r}(\theta, \phi)=\left[r_{i n} t_{r_{n}}^{2} t_{p_{n}}^{2}\left(j c_{n}\right)^{2} e^{j 2(n-1)\left(\alpha+\alpha_{s}\right)}\left(\prod_{i=1}^{n-1} t_{c_{i}} e^{j \psi}\right)^{2}\right] \text {, }
\end{aligned}
$$

where $r_{l}$ is the reflection coefficient at $n$th element terminated by a load impedance of $Z_{l_{n}}$ and $r_{i n}$ is the reflection coefficient 


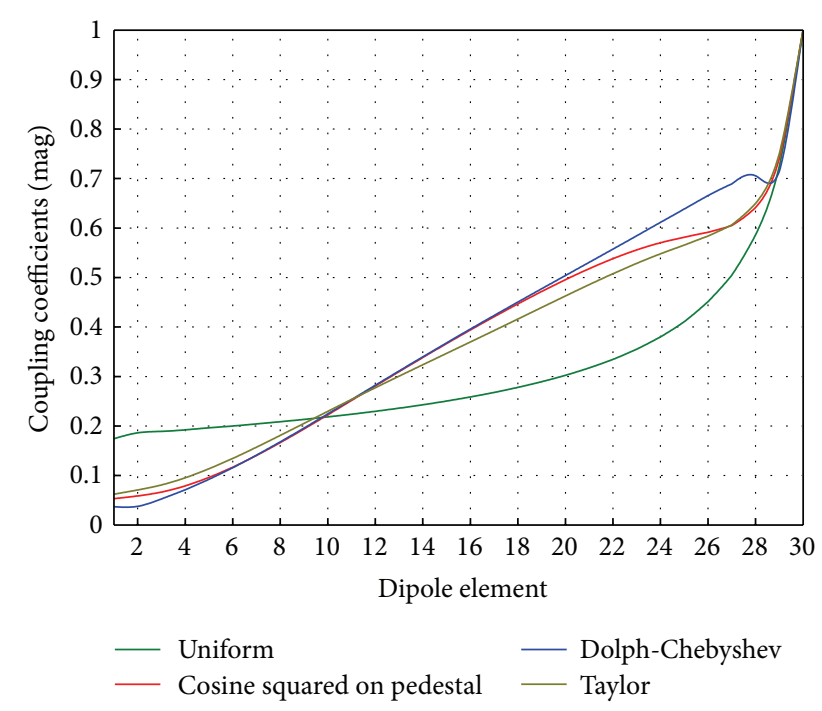

Figure 7: Coupling coefficients for different amplitude distributions in a series-fed linear dipole array; collinear configuration. $d=0.1 \lambda$.

at the receive port connected to a coaxial cable of impedance $Z_{0}$. Equation (10) signifies the importance of choosing appropriate value for the impedance terminating the coupler ports in the network. From this, the RCS due to the signal scattering beyond the coupling port of couplers becomes

$$
\begin{aligned}
& \sigma_{s}(\theta, \phi) \\
&=\mid \sum_{n=1}^{N}\left\{\frac { j \eta _ { o } } { 4 \lambda Z _ { a } } h ^ { 2 } \operatorname { c o s } \theta \left(\vec{E}_{1_{n}}^{r}(\theta, \phi)+\vec{E}_{2_{n}}^{r}(\theta, \phi)\right.\right. \\
&\left.\left.+\vec{E}_{3_{n}}^{r}(\theta, \phi)+\vec{E}_{4_{n}}^{r}(\theta, \phi)\right)\right\}\left.\right|^{2} .
\end{aligned}
$$

Thus, the total normalized RCS of the dipole array in the presence of mutual coupling, due to mismatches within the series-feed network, is given by

$$
\sigma(\theta, \phi)=\frac{4 \pi}{\lambda^{2}}\left\{\sigma_{r}(\theta, \phi)+\sigma_{p}(\theta, \phi)+\sigma_{c}(\theta, \phi)+\sigma_{s}(\theta, \phi)\right\}
$$

2.2. Parallel Feed Network. In a phased array with parallel feed network (Figure 9), the two input arms (Port 2 and Port 3 ) of a first level coupler are connected to two different elements and thus receive two different signals unlike the series feed network (Figure 9(a)). Amongst the other two ports, one acts as the sum port (Port 1) and the other as difference port (Port 4). Having intention of receiving maximum signal power, the difference ports are load terminated while the sum ports are connected to the input arms of the couplers in the further levels of the feed network.

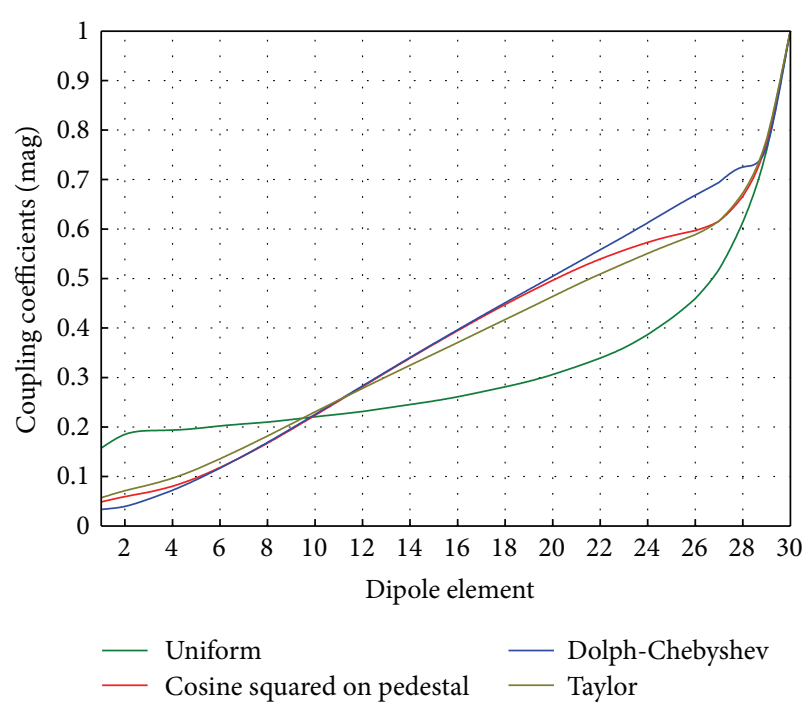

FIGURE 8: Coupling coefficients for different amplitude distributions in a series-fed linear dipole array; parallel-in-echelon configuration. $d=0.1 \lambda$.

In parallel-fed dipole array, the reflection coefficients at the input arms of the first-level couplers differ for the even and odd elements of the array. Mathematically,

$$
\begin{aligned}
& r_{c_{n}}=\left|\frac{Z_{33_{1 i}}-Z_{p_{n}}}{Z_{33_{1 i}}+Z_{p_{n}}}\right| \quad \text { for odd-numbered elements, } \\
& r_{c_{n}}=\left|\frac{Z_{22_{1 i}}-Z_{p_{n}}}{Z_{22_{1 i}}+Z_{p_{n}}}\right| \quad \text { for even-numbered elements, }
\end{aligned}
$$

where $i=1,2, \ldots,(N+1) / 2$ is an integer and $n=1,2, \ldots, N$.

The index $i$ increments at each odd element of the dipole array; that is,

$$
\begin{aligned}
r_{c_{1}} & =\left|\frac{Z_{33_{11}}-Z_{p_{1}}}{Z_{33_{11}}+Z_{p_{1}}}\right|, \\
r_{c_{2}} & =\left|\frac{Z_{22_{11}}-Z_{p_{2}}}{Z_{22_{11}}+Z_{p_{2}}}\right|, \\
r_{c_{3}} & =\left|\frac{Z_{33_{12}}-Z_{p_{3}}}{Z_{33_{12}}+Z_{p_{3}}}\right|, \\
& \vdots
\end{aligned}
$$

This yields the RCS contribution from the input ports of the first level couplers similar to that of series feed network. One can observe that the signal incident at the antenna passes through phase shifters and reaches the couplers. The 


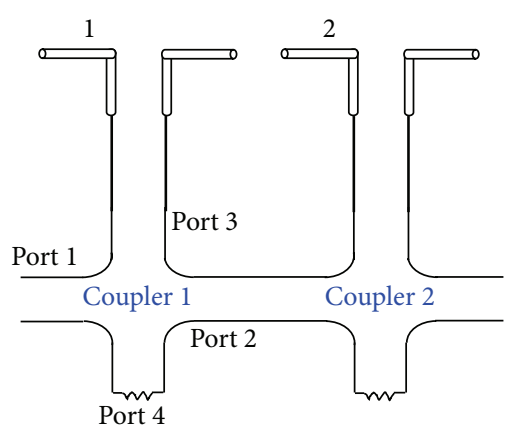

(a)

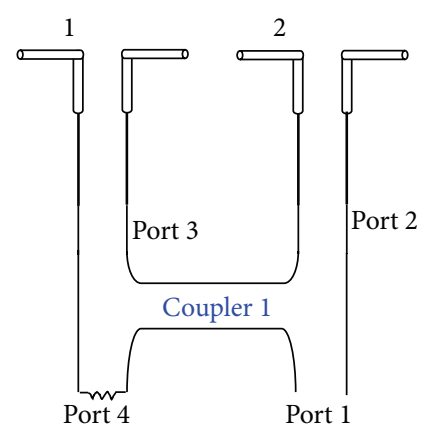

(b)

FIGURE 9: The arrangement of couplers. (a) Series feed network. (b) Parallel feed network.

signal, which manages to pass through the input arms of the couplers, would either move towards the sum or the difference port. At these points, significant reflection can occur due to the mismatch of impedances, indicated by the factors $r_{s_{q i}}$ and $r_{d_{q i}}$ for sum and difference ports, respectively (Figure 10). Here subsuffices $q$ and $i$ indicate the coupler-level and the coupler number. The magnitude of these reflection coefficients depends on both the position of dipole element in the array and the corresponding impedances.
2.2.1. First Level Couplers. Assuming all higher levels of couplers in the feed network, except for the first, to be perfectly matched, the scattered field can be calculated only for the first level of couplers. Hence, the characteristic impedance $\left(Z_{0}\right)$ at the receive port will appear at the sum ports of all first level couplers. The scattered field due to first level couplers at oddnumbered elements, owing to the signal reflections at sum and difference ports, obtained by following the path of the signal (Figure 11), is

$$
\vec{E}_{n_{\text {odd }}}^{r}(\theta, \phi)=t_{r_{n}} t_{p_{n}} e^{j(n-1)\left(\alpha+\alpha_{s}\right)}\left[\begin{array}{c}
r_{s_{(n / 2+1)}} c_{(n / 2+1)} e^{j \psi}\left\{t_{r_{n}} t_{p_{n}} e^{j(n-1)\left(\alpha+\alpha_{s}\right)} c_{(n / 2+1)} e^{j \psi}+t_{r_{n+1}} t_{p_{n+1}} e^{j(n)\left(\alpha+\alpha_{s}\right)} t_{c_{(n / 2+1)}}\right\} \\
+r_{d_{(n / 2+1)}} t_{\left.c_{(n / 2+1)}\right)}\left\{t_{r_{n}} t_{p_{n}} e^{j(n-1)\left(\alpha+\alpha_{s}\right)} t_{c_{(n / 2+1)}}+t_{r_{n+1}} t_{p_{n+1}} e^{j(n)\left(\alpha+\alpha_{s}\right)} c_{(n / 2+1)} e^{j \psi}\right\}
\end{array}\right] .
$$

Here the expressions for the sum and difference port reflection coefficients are given by

$$
\begin{aligned}
& r_{s_{1(n / 2+1)}}=\left|\frac{Z_{11_{1(n / 2+1)}}-Z_{13_{1(n / 2+1)}}}{Z_{11_{1(n / 2+1)}}+Z_{13_{1(n / 2+1)}}}\right| \\
& \vec{E}_{n_{\text {even }}}^{r}(\theta, \phi)=t_{r_{n}} t_{p_{n}} e^{j(n-1)\left(\alpha+\alpha_{s}\right)}\left[\begin{array}{c}
r_{s_{(n / 2)}} t_{c_{(n / 2)}}\left\{t_{r_{n-1}} t_{p_{n-1}} e^{j(n-2)\left(\alpha+\alpha_{s}\right)} c_{(n / 2)} e^{j \psi}+t_{r_{n}} t_{p_{n}} e^{j(n-1)\left(\alpha+\alpha_{s}\right)} t_{c_{n / 2)}}\right\} \\
+r_{\left.d_{(n / 2)}\right)} c_{(n / 2)} e^{j \psi}\left\{t_{r_{n-1}} t_{p_{n-1}} e^{j(n-2)\left(\alpha+\alpha_{s}\right)} t_{c_{(n / 2)}}+t_{r_{n}} t_{p_{n}} e^{j(n-1)\left(\alpha+\alpha_{s}\right)} c_{(n / 2)} e^{j \psi}\right\}
\end{array}\right],
\end{aligned}
$$

where

$$
\begin{gathered}
r_{d_{1(n / 2)}}=\left|\frac{Z_{44_{1(n / 2)}}-Z_{42_{1(n / 2)}} \mid}{Z_{44_{1(n / 2)}}+Z_{42_{1(n / 2)}}}\right|, \\
r_{s_{1(n / 2)}}=\left|\frac{Z_{22_{1(n / 2)}}-Z_{11_{1(n / 2)}}}{Z_{22_{1(n / 2)}}+Z_{11_{1(n / 2)}}}\right|,
\end{gathered}
$$

for even-numbered elements.
Thus, the RCS corresponding to the odd and even dipole elements in the array, due to reflections at sum and difference ports of couplers, is given by

$$
\begin{gathered}
\sigma_{\text {odd }}(\theta, \phi)=\left|\sum_{n=\text { odd }}^{N}\left\{\frac{j \eta_{o}}{4 \lambda Z_{a}} h^{2} \cos \theta \vec{E}_{n_{\text {odd }}}^{r}(\theta, \phi)\right\}\right|^{2}, \\
\sigma_{\text {even }}(\theta, \phi)=\left|\sum_{n=\text { even }}^{N}\left\{\frac{j \eta_{o}}{4 \lambda Z_{a}} h^{2} \cos \theta \vec{E}_{n_{\text {even }}}^{r}(\theta, \phi)\right\}\right|^{2} .
\end{gathered}
$$




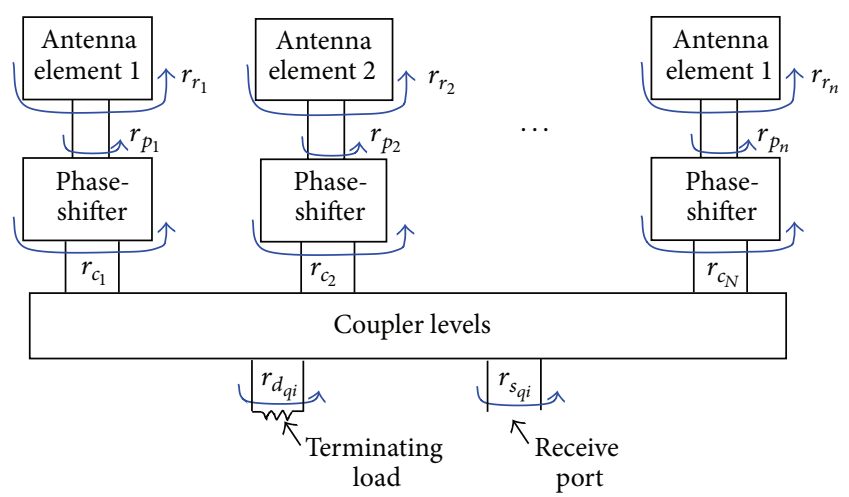

FIGURE 10: Reflection coefficients at different levels of the parallel feed network.

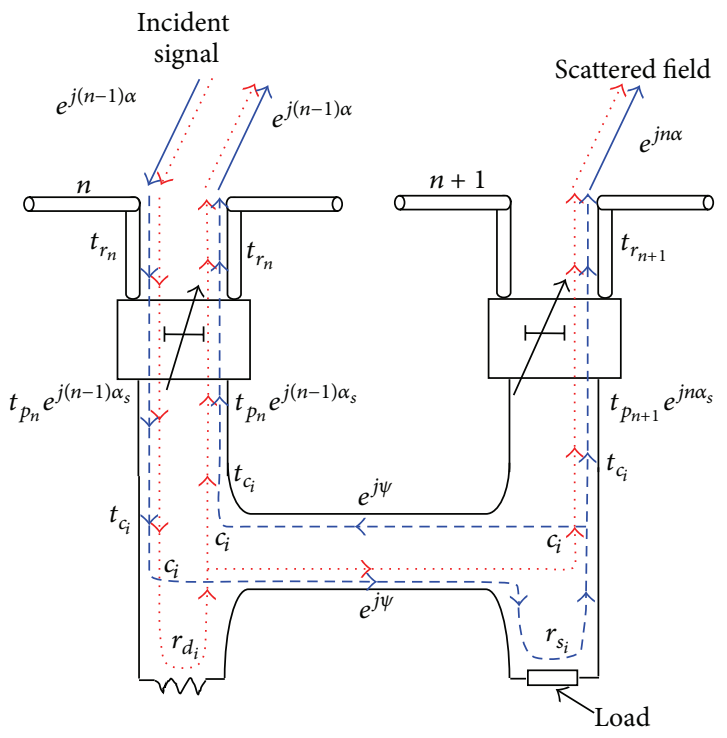

FIGURE 11: Signal paths through the first-level couplers in the parallel-feed network of a linear dipole array for $n$th odd-numbered element.

Thus, the normalized overall RCS of a parallel-fed linear dipole array, considering the scattering up to first level couplers, is expressed as

$$
\begin{array}{r}
\sigma(\theta, \phi)=\frac{4 \pi}{\lambda^{2}}\left\{\sigma_{r}(\theta, \phi)+\sigma_{p}(\theta, \phi)+\sigma_{c}(\theta, \phi)\right. \\
\left.+\sigma_{\text {odd }}(\theta, \phi)+\sigma_{\text {even }}(\theta, \phi)\right\} .
\end{array}
$$

\section{Results and Discussion}

In this paper, scattering from a linear dipole array fed by series and parallel feed network is calculated. The software code is developed in Fortran 90, based on the formulation discussed in Section 2. The RCS patterns are shown in plotting subroutine Sigmaplot v.10. The results are presented for both with and without mutual coupling effect. The current over the surface of each individual wire-type half wavelength dipole array is assumed to be a cosine function.

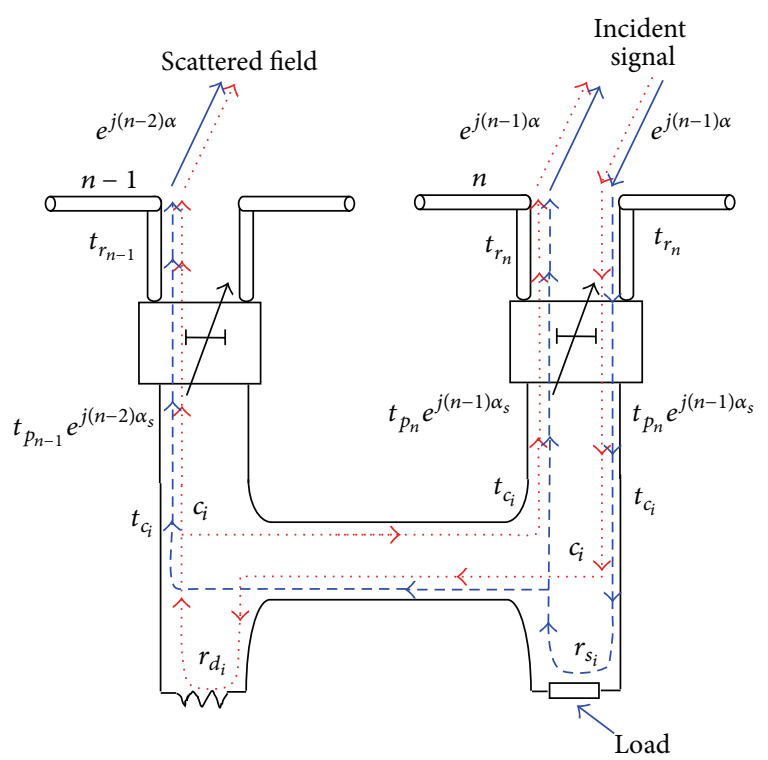

FIGURE 12: Signal paths through the first-level couplers in the parallel-feed network of a linear dipole array for $n$th even-numbered element.

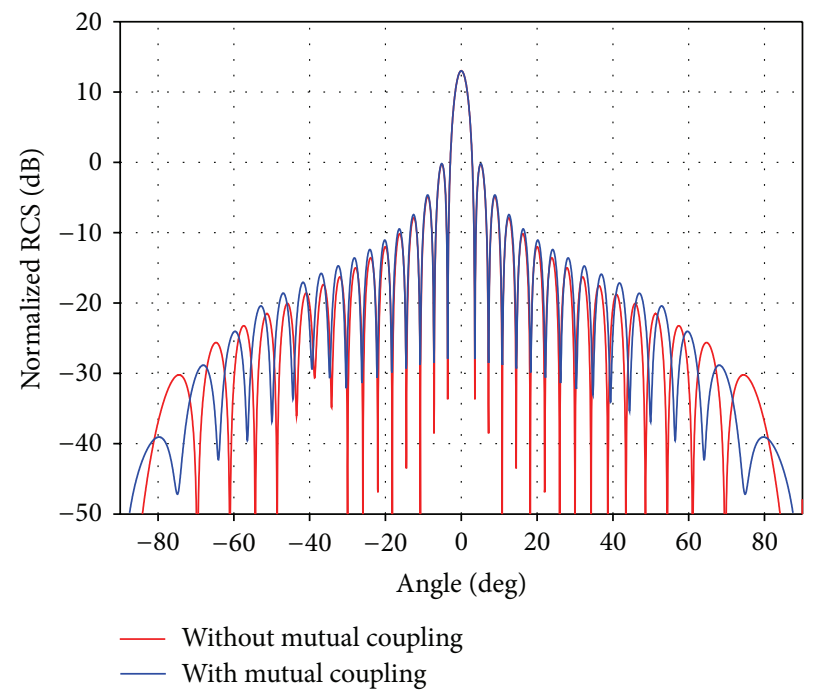

FIGURE 13: RCS of a parallel-in-echelon series-fed linear dipole array $(N=40)$ with Dolph-Chebyshev amplitude distribution $\left(\theta_{s}=0^{\circ}\right)$.

Figure 13 shows the broadside (at $0^{\circ}$ ) RCS of a seriesfed parallel-in-echelon 40-element dipole array, terminated by the load of $130 \Omega$. The receive port of the array system is connected to $100 \Omega$ coaxial cable. The amplitude distribution at the dipole feed terminals is taken to be Dolph-Chebyshev. The interelement spacing is taken as $0.2 \lambda$. It can be seen that in the RCS pattern, the specular lobe (lobe at $0^{\circ}$ ) remains the same for both with and without mutual coupling cases. However, a noticeable variation in the sidelobe location and levels is evident. The adverse effect of coupling on RCS pattern worsens as the array scan angle is increased from $0^{\circ}$ to $65^{\circ}$, keeping all other parameters unchanged (Figure 14). 


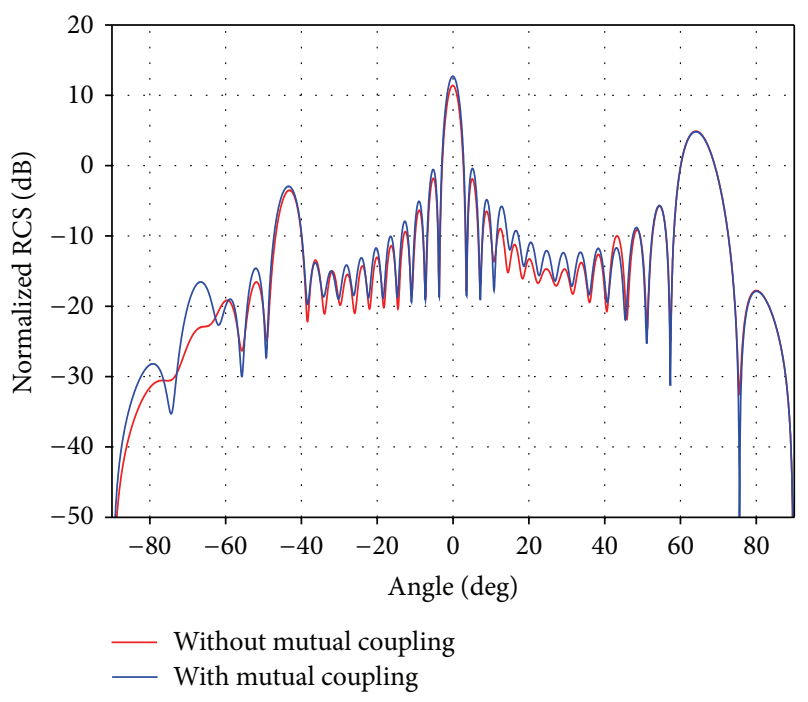

FIGURE 14: RCS of a series-fed parallel-in-echelon linear dipole array $(N=40)$ with Doplh-Chebyshev amplitude distribution $\left(\theta_{s}=65^{\circ}\right)$.

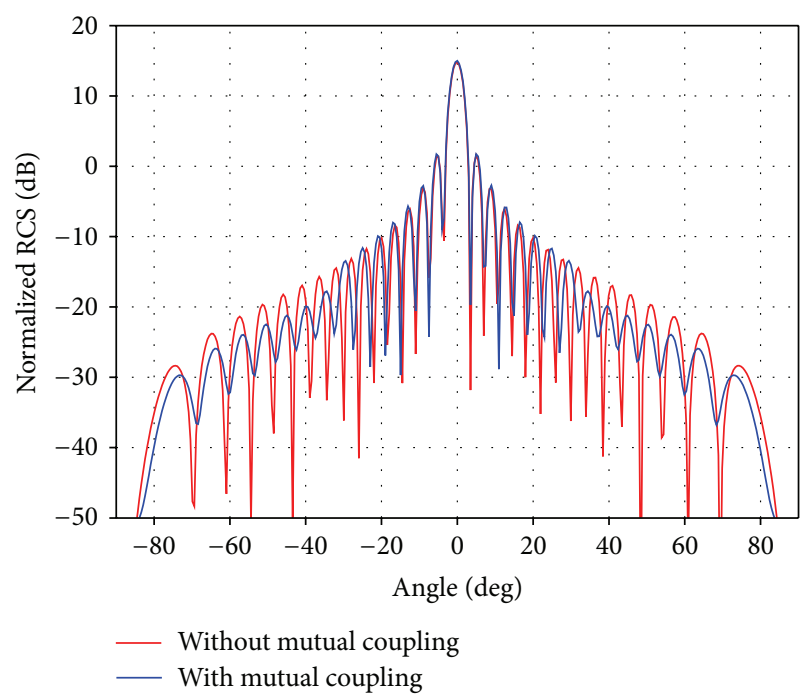

FIGURE 15: Broadside RCS of a series-fed 40-element linear dipole array in side-by-side configuration with Taylor amplitude distribution $\left(\theta_{\sigma}=0^{\circ}\right)$.

The observed phenomenon signifies the dependence of mutual coupling effect on the array scan angle [28].

Figures 15 and 16 exhibit a similar trend for 40-element linear dipole array in side-by-side configuration with Taylor amplitude distribution when terminated with $200 \Omega$. Results are compared for with and without mutual coupling cases.

The variation in the broadside RCS of a 32-element parallel-fed linear dipole array, with an interelement spacing of $0.4 \lambda$, in the presence of mutual coupling, is shown in Figure 17. Here all the couplers in the feed network are assumed to be equal power combiners (receive array), and the coupling and transmission coefficients are taken be $1 / \sqrt{2}$. The dipole elements are arranged in side-by-side configuration with $20 \Omega$ load termination at the difference arms of the first

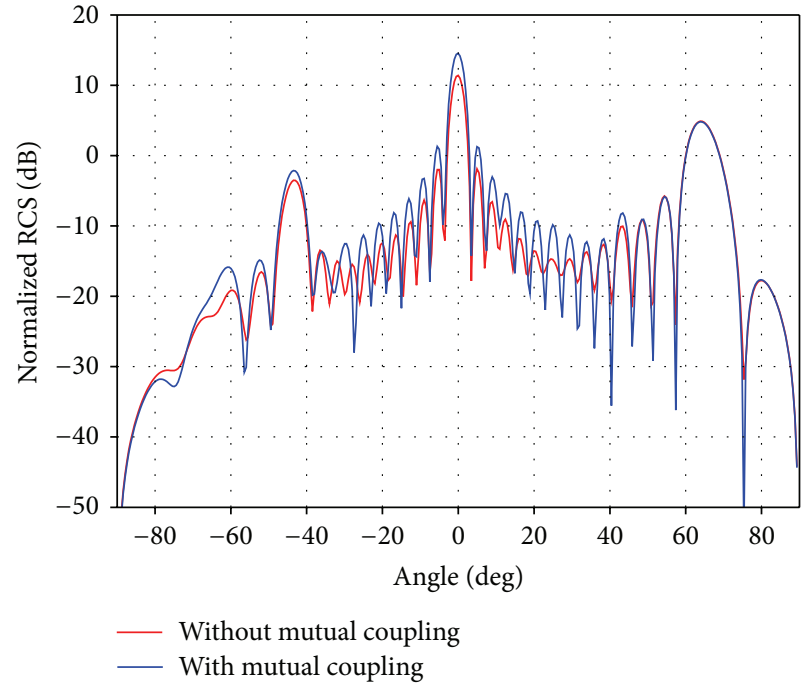

FIGURE 16: Scanned RCS of a series-fed 40-element linear dipole array in side-by-side configuration with Taylor amplitude distribution $\left(\theta_{\sigma}=65^{\circ}\right)$.

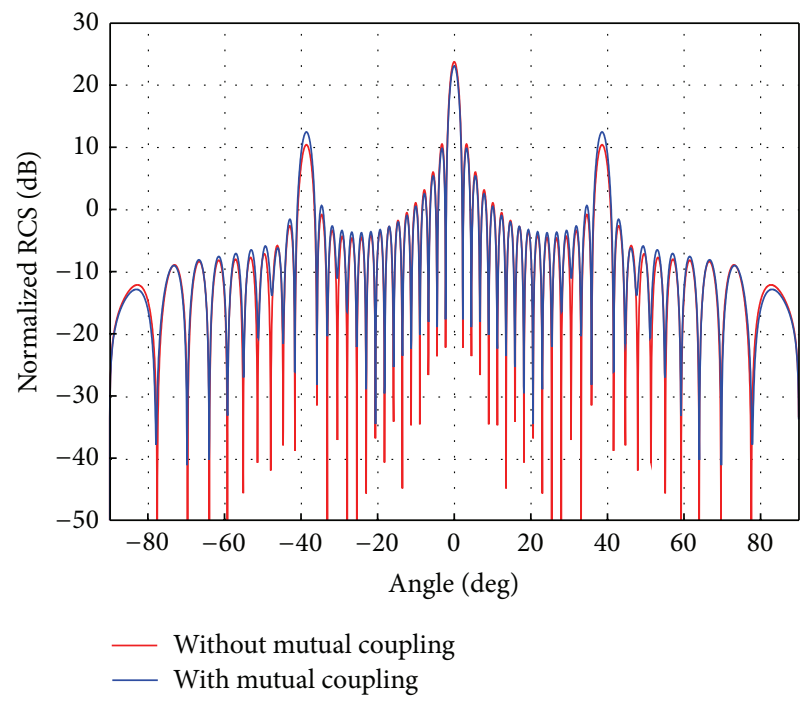

FIGURE 17: Broadside RCS of a parallel-fed 32-element linear dipole array in side-by-side configuration.

level couplers. A characteristic impedance of $50 \Omega$ is seen at the sum ports of all the couplers in the first level of feed network, when all higher levels are perfectly matched. It is seen that the RCS in the presence of coupling shows the specular lobe identical to that of no mutual coupling case. However, the level of the lobe at around $\pm 38^{\circ}$ is more as compared to no coupling case.

Figure 18 shows the scanned RCS (at $70^{\circ}$ ) of a 32-element parallel-fed dipole array arranged in collinear configuration, due to the scattering from first level couplers. An interelement spacing of $0.4 \lambda$ and uniform distribution with equal power combiners are assumed. Further, the terminating impedance at the difference arms of the couplers is taken to be $50 \Omega$, and the characteristic impedance is considered to be $85 \Omega$. 


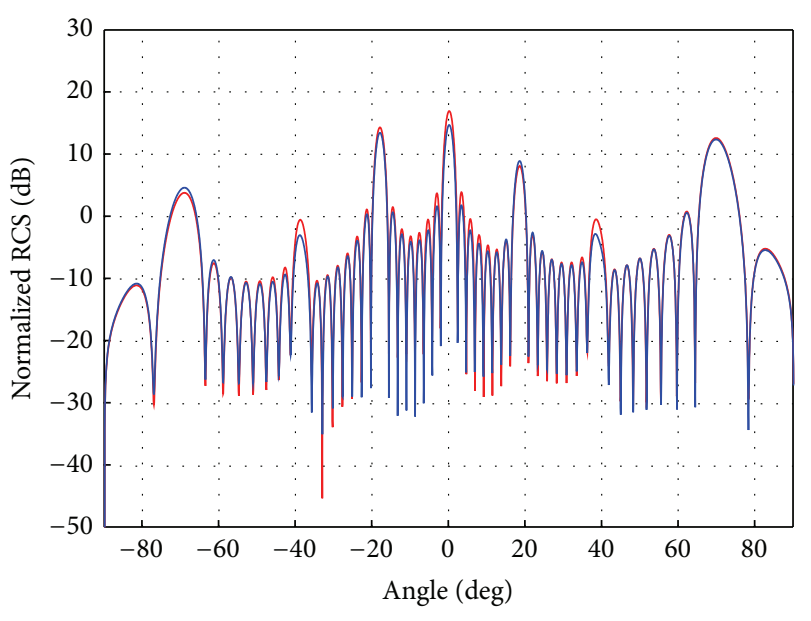

- Without mutual coupling

— With mutual coupling

FIGURE 18: Scanned RCS of a parallel-fed collinear 32-element dipole array $\left(\theta_{s}=70^{\circ}\right)$, due to the scattering at first-level couplers only.

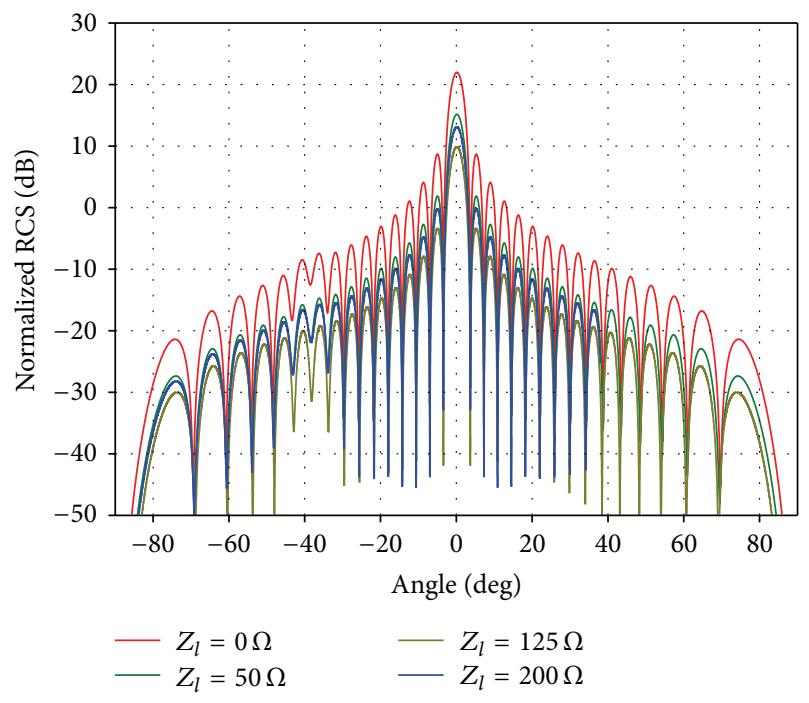

FIGURE 19: Effect of varying the impedance terminating the isolation port of couplers on the broadside RCS of a series-fed collinear dipole array in the presence of coupling.

Although the scanning is shown to reduce the level of the lobe at $0^{\circ}$ in the presence of mutual coupling, other lobes (at $+18^{\circ}$ and $-70^{\circ}$ ) are seen to rise as compared to no mutual coupling case.

Figure 19 shows the effect on the RCS due to the variation of load impedance terminating the isolation port of the couplers in a series feed network. A cosine squared on a pedestal distribution is assumed to excite series-fed 40element linear dipole array in collinear configuration. Other parameters are taken as $d=0.2 \lambda$ and $Z_{0}=100 \Omega$. It is observed that the RCS of a dipole array will be minimum when terminated with suitable impedance rather than when it is short. In other words, the RCS of the dipole array is seen

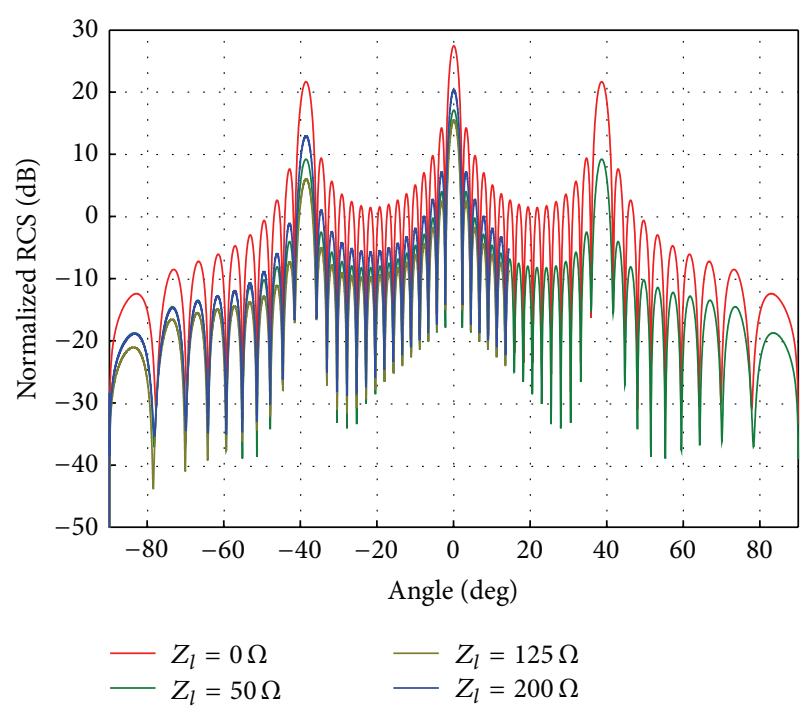

FIGURE 20: Effect of varying the impedance terminating the difference port of the first-level couplers on the broadside RCS of a parallel-fed linear 32-element dipole array.

to decrease as the impedance at the terminating ports of its couplers increases.

This trend is, however, seen to possess a limit, beyond which an inverse effect is observed; that is, as the value of terminating load impedance is increased, the scattered field and, hence, the RCS are increased.

The value of this limiting impedance can be termed as optimum impedance. For this impedance value, the RCS of the dipole array will be minimum. Thus, one can infer that the RCS of a phased array can be optimized using variable terminating impedance for a given spatial geometry, feed network, and amplitude distribution. This is further evident from Figure 20. It shows the effect of varying the terminating impedance on the RCS pattern of a 32-element parallel-inechelon dipole array with parallel feed network (at the first level of the couplers). The presence of mutual coupling is taken into account.

\section{Conclusions}

This paper provides an insight into the radar cross-section (RCS) of series and parallel-fed linear dipole arrays in the presence of mutual coupling. The formulation for the scattered field at the levels of feed network is presented in terms of array design parameters like the terminating loads, characteristic impedance, and the reflection coefficients of the feed network including the mutual coupling factor. The formulation for scattered fields in both series and parallelfed linear arrays is identical up to the level of couplers owing to the similarities in their schematic. However, formulations diverge in that the series feed network is in terms of signal path, whereas in the parallel feed network, the formulation depends not only on signal path but also on the position of the array element (even or odd). The scattered field at each component level is coherently summed to arrive at the total RCS of the phased array. 
The simulation results presented in this paper demonstrate the dependence of RCS of the dipole array on the mutual coupling, especially the scanned RCS. However, the trend of RCS variation due to the change in amplitude distribution and array configuration is seen to be almost independent of the feed network for both with and without mutual coupling. Further, the role of the optimum terminating impedance in controlling the total RCS of dipole array is shown for both types of the feed network. It is inferred that an array with variable terminal impedance can contribute towards the RCS control of phased array. This technique of conforming to the signal path to arrive at the RCS of the phased array is extendable to an array of arbitrary size and for multiple coupler levels (for parallel-feed or arbitrary feed). The results presented are applicable while designing a low observable phased array for optimum performance.

\section{List of Symbols}

$\alpha: \quad$ Interelement space delay along $x$-direction

$\alpha_{s}: \quad$ Interelement phase to scan antenna beam

$\begin{array}{ll}\Gamma_{n}^{r}: & \text { along } x \text {-direction } \\ & \text { Total reflected signal returning to aperture }\end{array}$ element $n$

$\eta$ : $\quad$ Impedance of medium around the antenna

$\eta_{0}: \quad$ Impedance of free space

$(\theta, \phi): \quad$ Direction of incident signal

$\left(\theta_{s}, \phi_{s}\right): \quad$ Beam scan angle

$\lambda: \quad$ Wavelength

$\sigma(\theta, \phi): \quad$ Overall RCS of phased array

$\sigma_{c}(\theta, \phi): \quad$ RCS due to scattering at the junction of phase-shifters and the coupler arm(s)

$\sigma_{\text {even }}(\theta, \phi)$ : RCS corresponding to even dipole elements in the array

$\sigma_{\text {odd }}(\theta, \phi)$ : RCS corresponding to odd dipole elements in the array

$\sigma_{p}(\theta, \phi): \quad$ RCS due to scattering from phase-shifters

$\sigma_{r}(\theta, \phi): \quad$ RCS due to scattering from radiators

$\sigma_{s}(\theta, \phi): \quad$ RCS due to scattering beyond the coupling port of couplers

$c_{n}: \quad$ Coupling coefficient of $n$th coupler arm

$d$ : $\quad$ Interelement spacing along $x$-axis

$\vec{d}_{n}: \quad$ Distance vector

$\vec{E}^{i}(\theta, \phi): \quad$ Incident field

$\vec{E}_{c_{n}}^{r}(\theta, \phi): \quad$ Scattered field due to the coupling/ input port(s) of $n$th coupler

$\vec{E}_{n}^{r}(\theta, \phi): \quad$ Total scattered field

$\vec{E}_{n_{\text {even }}}^{r}(\theta, \phi)$ : Scattered field at $n$th even-numbered element due to first-level couplers

$\vec{E}_{n_{\text {odd }}}^{r}(\theta, \phi)$ : Scattered field at $n$th odd-numbered element due to first-level couplers

$\vec{E}_{p_{n}}^{r}(\theta, \phi): \quad$ Scattered field due to $n$th phase-shifter

$\vec{E}_{r_{n}}^{r}(\theta, \phi): \quad$ Scattered field due to $n$th dipole

$\vec{E}_{1_{n}}^{r}(\theta, \phi)$ : Scattered field due to forward traveling wave at $n$th element

$\vec{E}_{2_{n}}^{r}(\theta, \phi): \quad$ Scattered field due to backward traveling wave at $n$th element
$\vec{E}_{3_{n}}^{r}(\theta, \phi)$ : Scattered field due to self-reflected wave at $n$th element

$\vec{E}_{4_{n}}^{r}(\theta, \phi)$ : Scattered field due to input-load reflected wave at $n$th element

$\vec{E}_{n}^{s}$ : $\quad$ Total scattered field due to single element

$\vec{h}$ : $\quad$ Effective height of $x$-polarized antenna

$i$ : $\quad$ Coupler number

$i_{n}$ : $\quad$ Current at the feed terminals of the $n$th

antenna element

$k: \quad$ Wave number

$\vec{k}: \quad$ Wave vector

$L_{n}: \quad$ Length of delay-lines

$N$ : $\quad$ Number of elements in the antenna array

q: $\quad$ Coupler level

$r_{c_{n}}$ : Reflection coefficient at the junction of phaseshifter and the $n$th coupler

$r_{d_{q i}}$ : Reflection coefficient at the difference port of $i$ th coupler in $q$ th level

$r_{i n}$ : Reflection coefficient at the receive port of the array

$r_{l_{n}}: \quad$ Reflection coefficient of the load terminating the port of $n$th coupler

$r_{p_{n}}: \quad$ Reflection coefficient of $n$th phase-shifter

$r_{r_{n}}: \quad$ Reflection coefficient of $n$th radiating element

$r_{s_{q i}}: \quad$ Reflection coefficient at the sum port of $i$ th coupler in $q$ th level

$R: \quad$ Distance between the target and the observation point

$t_{c_{n}}: \quad$ Transmission coefficient of $n$th coupler

$t_{p_{n}}: \quad$ Transmission coefficient of $n$th phase-shifter

$t_{r_{n}}: \quad$ Transmission coefficient of $n$th radiator

$Z_{0}: \quad$ Characteristic impedance

$Z_{a}: \quad$ Antenna impedance

$Z_{a_{n}}: \quad$ Terminal impedance of $n$th antenna element

$Z_{c_{n}}$ : Impedance at the input/coupling port of the couplers

$Z_{l_{n}}: \quad$ Load terminating isolation port of $n$th coupler

$Z_{l_{q i}}: \quad$ Load terminating the difference port of $i$ th coupler in $q$ th level

$Z_{m m_{q i}^{\prime}}$ : Impedance at the port $m$ and port $m^{\prime}$ of $i$ th coupler in $q$ th level

$Z_{p_{n}}: \quad$ Terminal impedance of $n$th phase-shifter.

\section{Acknowledgment}

This work was carried out under the Grant-in-Aid Aeronautics Research and Development Board (AR\&DB) Project no. A-1-178 at CSIR-National Aerospace Laboratories (NAL), Bangalore, India.

\section{References}

[1] C. Bourlier and P. Pouliguen, "Useful analytical formulae for near-field monostatic radar cross section under the physical optics: far-field criterion," IEEE Transactions on Antennas and Propagation, vol. 57, no. 1, pp. 205-214, 2009.

[2] W. Wiesbeck and E. Heidrich, "Influence of antennas on the radar cross section of camouflaged aircraft," in Proceedings of 
the 9th International Conference on Ground Penetrating Radar, pp. 122-125, Brighton, UK, October 1992.

[3] B. Thors and L. Josefsson, "Radiation and scattering tradeoff design for conformal arrays," IEEE Transactions on Antennas and Propagation, vol. 51, no. 5, pp. 1069-1076, 2003.

[4] W. T. Wang, Y. Liu, S. X. Gong, Y. J. Zhang, and X. Wang, "Calculation of antenna mode scattering based on method of moments," Progress In Electromagnetics Research Letters, vol. 15, pp. 117-126, 2010.

[5] A. Zdunek and W. Rachowicz, "Cavity radar cross section prediction," IEEE Transactions on Antennas and Propagation, vol. 56, no. 6, pp. 1752-1762, 2008.

[6] W. T. Wang, S. X. Gong, Y. J. Zhang, F. T. Zha, J. Ling, and T. T. Wan, "Low RCS dipole array synthesis based on MoMPSO hybrid algorithm," Progress in Electromagnetics Research, vol. 94, pp. 119-132, 2009.

[7] X. Li and Z. P. Nie, "Mutual coupling effects on the performance of MIMO wireless channels," IEEE Antennas and Wireless Propagation Letters, vol. 3, no. 1, pp. 344-347, 2004.

[8] R. Goossens and H. Rogier, "A hybrid UCA-RARE/RootMUSIC approach for 2-D direction of arrival estimation in uniform circular arrays in the presence of mutual coupling," IEEE Transactions on Antennas and Propagation, vol. 55, no. 3, pp. 841-849, 2007.

[9] D. C. Jenn and S. Lee, "Inband scattering from arrays with series feed networks," IEEE Transactions on Antennas and Propagation, vol. 43, no. 8, pp. 867-873, 1995.

[10] D. C. Jenn and V. Flokas, "In-band scattering from arrays with parallel feed networks," IEEE Transactions on Antennas and Propagation, vol. 44, no. 2, pp. 172-178, 1996.

[11] B. K. Lau, J. B. Andersen, G. Kristensson, and A. F. Molisch, "Impact of matching network on bandwidth of compact antenna arrays," IEEE Transactions on Antennas and Propagation, vol. 54, pp. 3225-3238, 2006.

[12] J. C. Coetzee and Y. Yu, "Port decoupling for small arrays by means of an eigenmode feed network," IEEE Transactions on Antennas and Propagation, vol. 56, no. 6, pp. 1587-1593, 2008.

[13] H. S. Lui, H. T. Hui, and M. S. Leong, "A note on the mutualcoupling problems in transmitting and receiving antenna arrays," IEEE Antennas and Propagation Magazine, vol. 51, no. 5, pp. 171-176, 2009.

[14] I. J. Gupta and A. A. Ksienski, "Effect of mutual coupling on the performance of adaptive arrays," IEEE Transactions on Antennas and Propagation, vol. 31, no. 5, pp. 785-791, 1983.

[15] K. M. Pasala and E. M. Friel, "Mutual coupling effects and their reduction in wideband direction of arrival estimation," IEEE Transactions on Aerospace and Electronic Systems, vol. 30, no. 4, pp. 1116-1122, 1994.

[16] R. S. Adve and T. K. Sarkar, "Compensation for the effects of mutual coupling on direct data domain adaptive algorithms," IEEE Transactions on Antennas and Propagation, vol. 48, no. 1 , pp. 86-94, 2000.

[17] B. Friedlander and A. J. Weiss, "Direction finding in the presence of mutual coupling," IEEE Transactions on Antennas and Propagation, pp. 273-284, 1991.

[18] K. R. Dandekar, H. Ling, and G. Xu, "Experimental study of mutual coupling compensation in smart antenna applications," IEEE Transactions on Wireless Communications, vol. 1, no. 3, pp. 480-487, 2002.

[19] H. T. Hui, "A practical approach to compensate for the mutual coupling effect in an adaptive dipole array," IEEE Transactions on Antennas and Propagation, vol. 52, no. 5, pp. 1262-1269, 2004.
[20] H. T. Hui, "A new definition of mutual impedance for application in dipole receiving antenna arrays," IEEE Antennas and Wireless Propagation Letters, vol. 3, no. 1, pp. 364-367, 2004.

[21] H. S. Lui and H. T. Hui, "Improved mutual coupling compensation in compact antenna arrays," IET Microwaves, Antennas and Propagation, vol. 4, no. 10, pp. 1506-1516, 2010.

[22] H. S. Lui and H. T. Hui, "Mutual coupling compensation for direction-of-arrival estimations using the receiving-mutualimpedance method," International Journal of Antennas and Propagation, vol. 2010, Article ID 373061, 7 pages, 2010.

[23] C. H. Niow, Y. T. Yu, and H. T. Hui, "Compensate for the coupled radiation patterns of compact transmitting antenna arrays," IET Microwaves, Antennas and Propagation, vol. 5, no. 6, pp. 699704, 2011.

[24] B. H. Wang and H. T. Hui, "Wideband mutual coupling compensation for receiving antenna arrays using the system identification method," IET Microwaves, Antennas and Propagation, vol. 5, no. 2, pp. 184-191, 2011.

[25] H. L. Sneha, Hema Singh, and R. M. Jha, "Mutual coupling effects for radar cross section (RCS) of a series-fed dipole antenna array," International Journal of Antennas and Propagation, vol. 2012, Article ID 601532, 20 pages, 2012.

[26] C. A. Balanis, Antenna Theory, Analysis and Design, John Wiley \& Sons, New Jersey, NY, USA, 2005.

[27] J. L. Volakis, Antenna Engineering Handbooked, McGraw Hill, New York, NY, USA, 4th edition, 2007.

[28] K. M. Lee and R. S. Chu, "Analysis of mutual coupling between a finite phased array of dipoles and its feed network," IEEE Transactions on Antennas and Propagation, vol. 36, no. 12, pp. 1681-1699, 1988. 

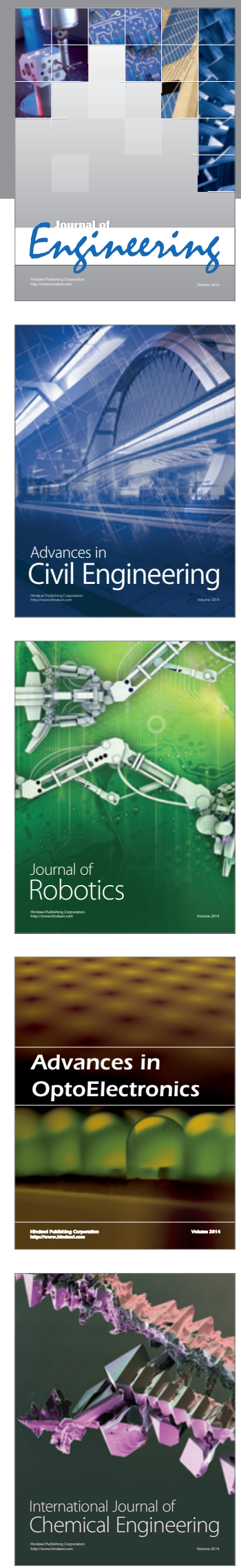

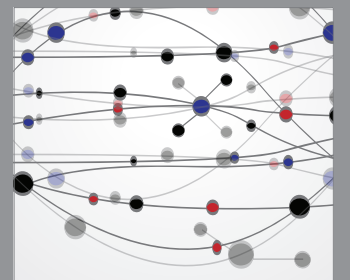

The Scientific World Journal
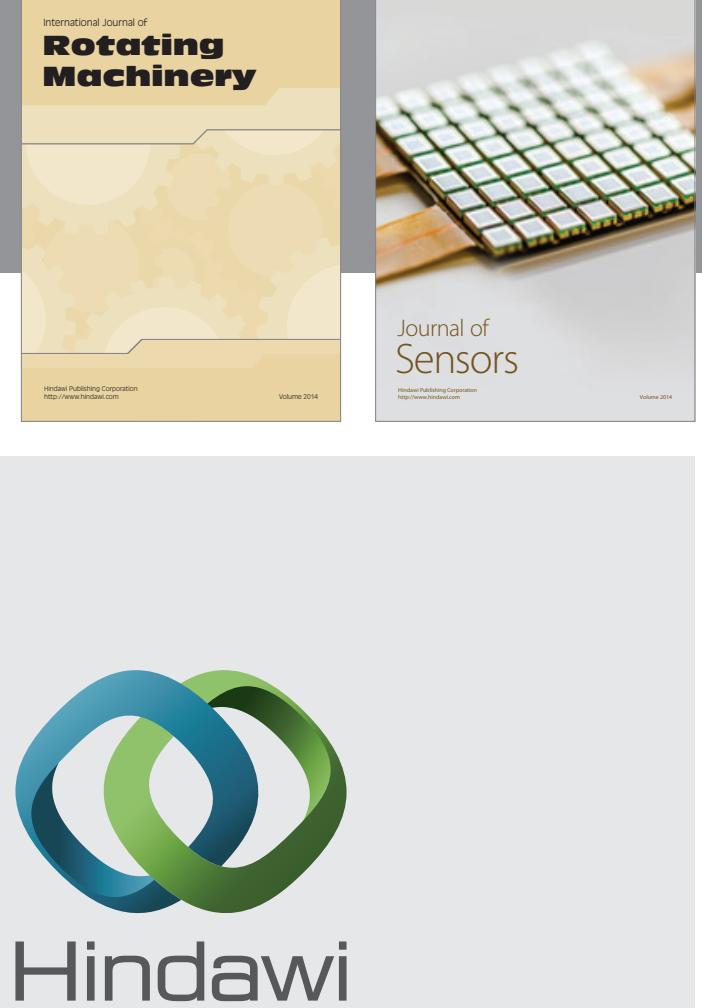

Submit your manuscripts at http://www.hindawi.com
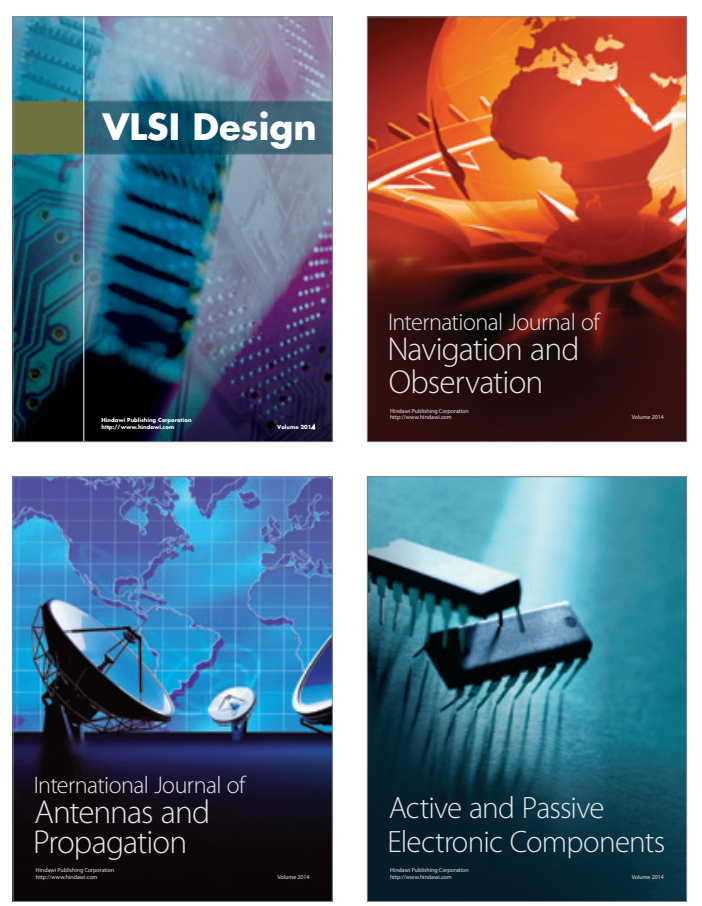
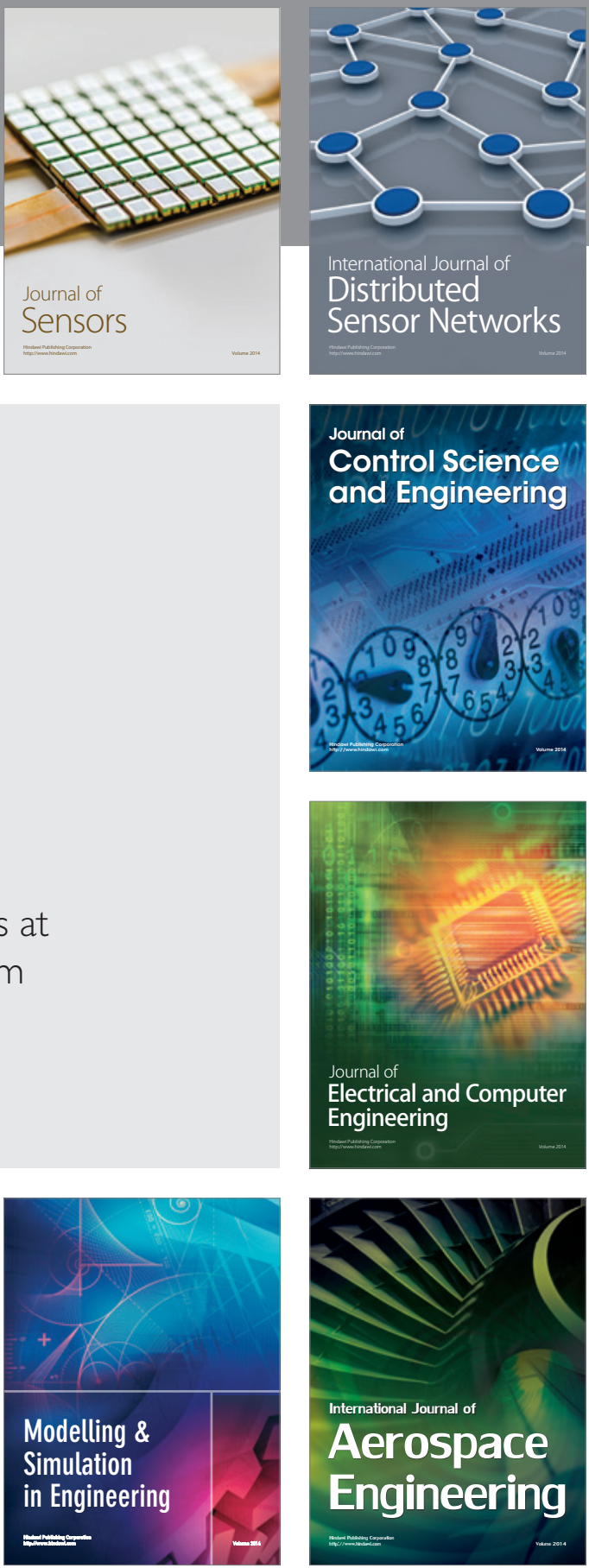

Journal of

Control Science

and Engineering
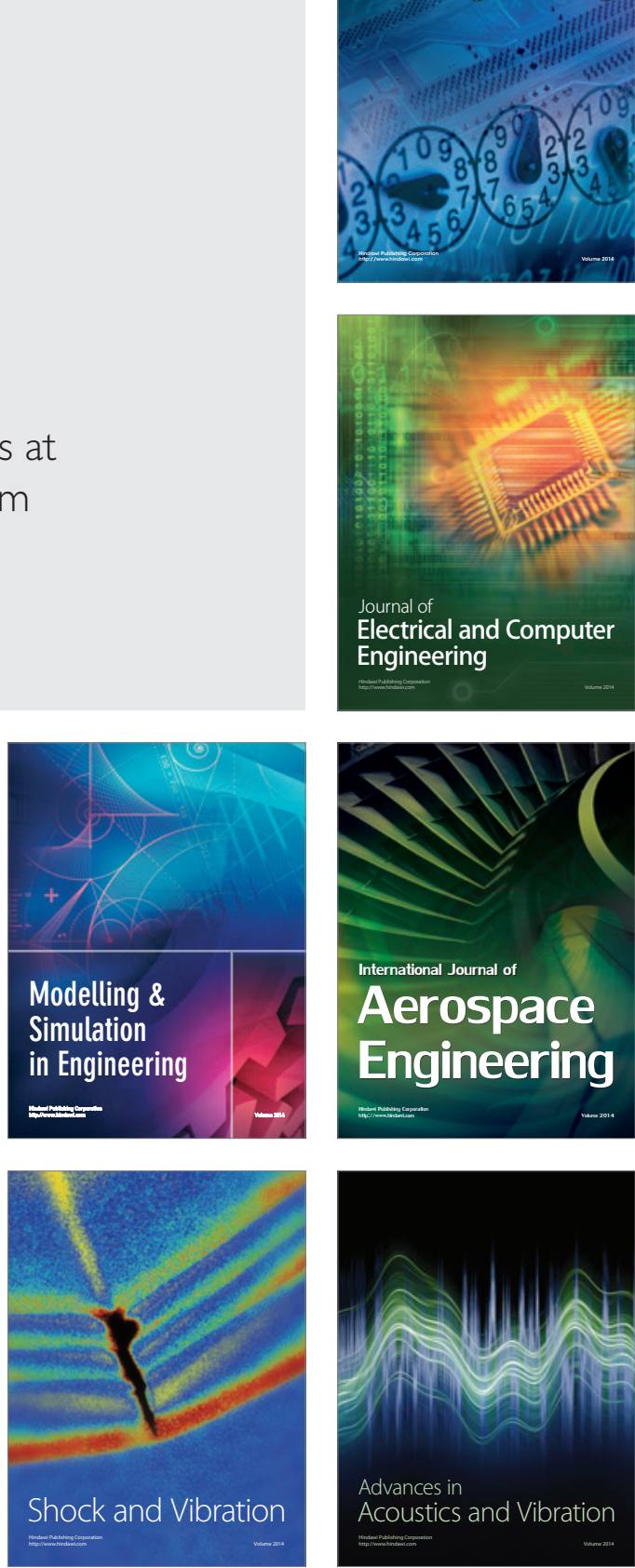